\title{
En torno a los bienes comunales. Posesiones y propiedades familiares con responsabilidades públicas en Rumanía
}

\author{
Míriam Torrens Arnal ${ }^{1}$ \\ Recibido: 9 de febrero de 2017/Aceptado: 31 de marzo de 2017
}

Resumen. Este artículo se propone reflexionar sobre los valores de justicia y equidad presentes en una institución consuetudinaria propia de la minoría székely de Transilvania, de habla húngara. El közbirtoksság (literalmente y simplificando: co-propiedad) se compone de propiedades familiares que constituyen un comunal agregado, es decir, una propiedad conjunta de pastos y bosque. Son indivisibles, inalienables y de gestión participativa directa y completa. Nos interesa subrayar que los comunales no son instituciones aisladas de una sociedad, sino que son coherentes con muchas otras instituciones locales y valores de esa misma sociedad y que conforman un modelo de convivencia particular. Veremos que entre el acuerdo y la tensión ocurren varios conflictos de intereses entre sus miembros. Junto a ellos, los intereses del Estado y de la administración local marcan la evolución del conjunto de sus regulaciones a lo largo de la historia.

Palabras clave: Comunales; Székely; Közbirtoksság; Relaciones de Propiedad.

\section{[en] Communal goods. Possessions and family properties with public respon- sibilities in Romania}

\begin{abstract}
The aim of this article is to focus on the values of justice and equity present in a customary institution of a sekler minority of Transylvania (Romania). The közbirtoksság is composed by family properties, constituting a joint property of pastures and forests. They are inalienable, indivisible and managed by a participatory and direct system. We would like to emphasize that the commons are not isolated institutions of a society, but on the contrary, they keep coherence and synergy with other local institutions and values. They conform a particular pattern of social living. Also, both State agencies and Local administration intervene at different historical conjunctures to the general pattern of evolution of the Commons.
\end{abstract}

Keywords: Commons; Székely; Közbirtoksság; Property relations.

Sumario. Contextualización. La propiedad como base del modelo social. Otras instituciones de asociación y cooperación de carácter comunal. El közbirtoksság: origen y tipologías. Historia reciente del közbirtoksság. Reglas de equidad y conflictos en el közbirtkosság. Conclusiones. Bibliografía.

Cómo citar: Torrens Arnal, M. (2017). En torno a los bienes comunales. Posesiones y propiedades familiares con responsabilidades públicas en Rumanía, en Revista de Antropología Social 26(2), 217-234.

It is too easy, and a serious mistake, to romanticize these social arrangements that distinguish much of peasant society. They are not radically egalitarian. Rather, they imply only that all are entitled to a living out of the resources within the village (...). (Scott, 1976: 5)

$1 \quad$ Universidad Autónoma de Barcelona.miriamtorrens@yahoo.es 


\section{Contextualización}

El artículo que se presenta parte del trabajo de campo etnográfico realizado en una comunidad rural situada en los altiplanos orientales de Transilvania, entre 600 y 700 metros de altitud ${ }^{2}$. Es un pueblo de pequeños propietarios que viven de una economía de subsistencia que combina agricultura, ganadería y explotación forestal. Respecto a las características demográficas, se trata de una población muy envejecida que suma unos 300 habitantes, aunque ha llegado a contar 1600 habitantes. La caída demográfica brusca coincide con el periodo socialista, especialmente el periodo de colectivización del suelo agrario y forestal, entre 1956 y 1962, y se mantiene en el periodo post-socialista. Las infraestructuras del pueblo son realmente deficitarias y cuenta con muy mala comunicación con el exterior.

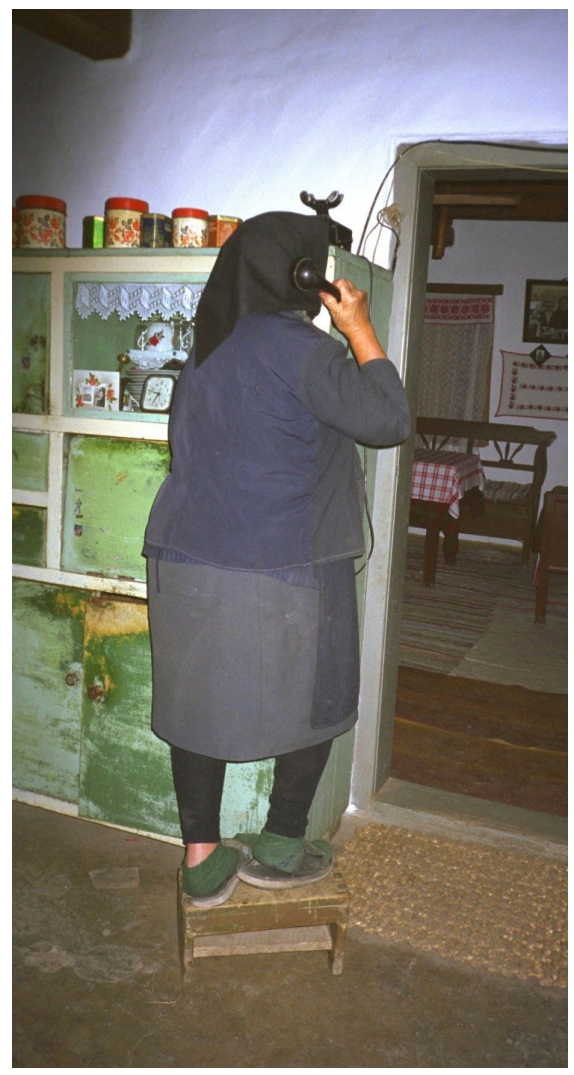

Fotografía 1. El único teléfono que había en el pueblo en el año 2000 no tenía línea directa, funcionaba a través de la centralita de un pueblo cercano. No había cobertura para móviles.

La población pertenece a la minoría székely3 de Rumania. La llamada tierra székely, Székelyföld, la componen los condados de Hargita, Covasna y Mures donde los húngaro-hablantes representan en torno al $90 \%$ de la población.

2 La etnografía realizada fue la base de la tesis doctoral presentada en la Universidad Autónoma de Barcelona con el título Pagesos del Post-socialisme. Un estudi de comunitat a Transilvània. Ver Torrens (2013).

3 El origen de esta población es todavía objeto de debate, pero lo que parece históricamente relevante es que su posición fronteriza, ya que Transilvania era una frontera geográfica y militar del Reino de Hungría, los definió como una población que proporcionaba un servicio militar a la corona húngara desde el siglo XII. Como reco- 
Los datos etnográficos del trabajo de campo utilizados corresponden a diversas estancias de varios meses realizadas entre los años 2000 y 2010. La estrategia principal de investigación consistió en la inmersión en la vida de la comunidad. Durante esta convivencia continuada se utilizaron técnicas que caracterizan el trabajo de campo antropológico: observación participante, entrevistas, genealogías, trabajo con documentos personales, vaciado de datos catastrales, de censos y del archivo parroquial.

La forma de aproximación a esta comunidad ha sido la de entenderla como un complejo humano construido para convivir. Se trata de un sistema de convivencia que va más allá de una unidad administrativa y que se distingue por sus valores, por sus medios de subsistencia, por formas de derecho consuetudinario, por sus instituciones y por las formas relacionales que se establecen entre sus miembros. Así, la pertenencia a la comunidad implica un compromiso practicado de un modelo de convivencia. Esta pertenencia a la comunidad es lo que otorga el derecho a los bienes comunales, los pastos, los bosques y la tierra. Es también este compromiso convivencial el que establece obligaciones relacionadas con la reciprocidad y la responsabilidad social.

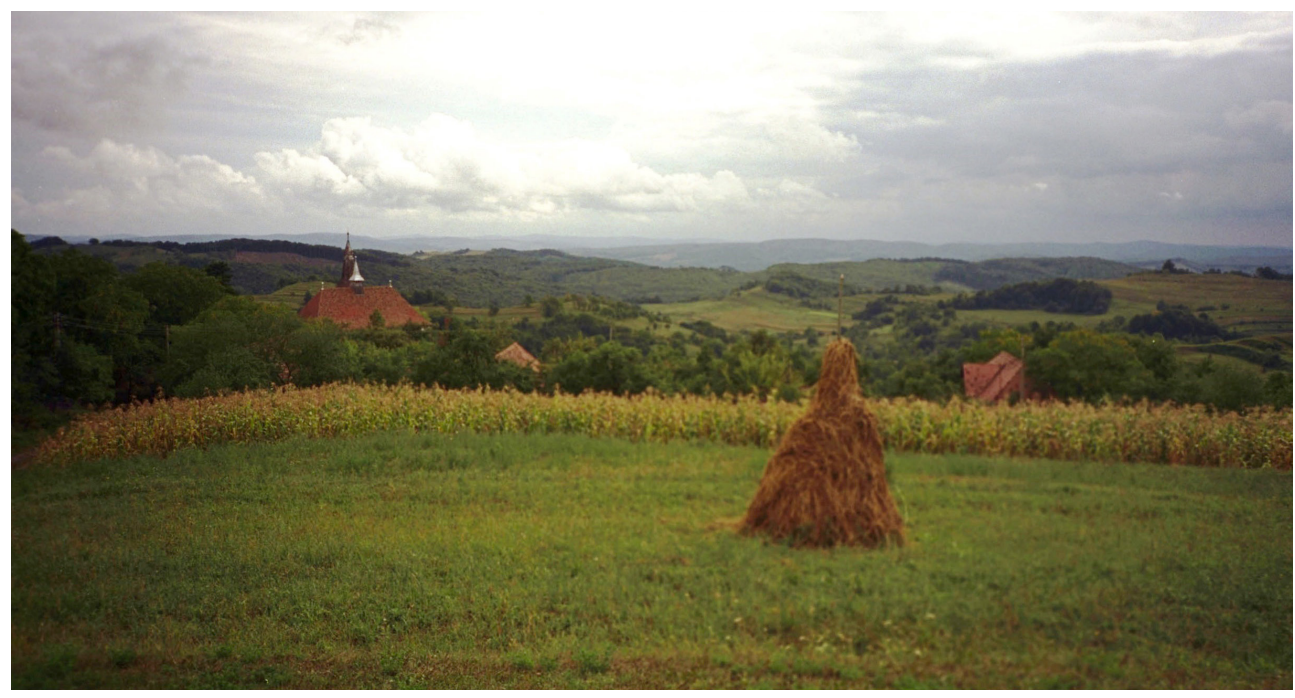

Fotografía 2. Imagen de los campos que rodean el pueblo

\section{La propiedad como base del modelo social}

Las reformas en el sistema de propiedad han sido el elemento central tanto de los proyectos socialistas como liberales para alcanzar los modelos de sociedad que defendían. Durante el período socialista, el Estado expropió el közbirtoksság a sus propietarios. A lo largo de este artículo iremos caracterizando el közbirtoksság. Se trata básicamente de una institución que gestiona conjuntamente propiedades fa-

nocimiento de esta función gozaron de un estatus muy especial, eran hombres libres que no tenían obligaciones de vasallaje ni de servitud. Recordemos también que Transilvania formó parte del Imperio austrohúngaro hasta después de la primera guerra mundial, cuando queda anexionada a Rumania por el Tratado de Trianon (1920) y, por lo tanto, los székely pasaron a constituir una minoría de habla húngara en Rumanía. 
miliares de pastos y bosques y que asume responsabilidades públicas de la vida en la comunidad. El periodo post-socialista, en cambio, se definió por el consenso respecto a la necesidad de un proceso radical de privatización, y el Estado ofreció la posibilidad de dividir el közbirtoksság en parcelas individuales. En esta coyuntura, la comunidad decidió por votación restablecer los antiguos estatutos consuetudinarios del közbirtoksság. Ello impidió la expropiación de la capacidad de control, explotación y gestión de la tierra y sus frutos, garantizándose el derecho a la seguridad de subsistencia (Scott, 1976).

En el campo de la antropología dedicada al post-socialismo europeo se ha vivido un resurgimiento del interés por la propiedad (Hann, 2003, 2005, 2006; Verdery, 2003, 2001; Kaneff, 2000; Creed, 1998; Cartwright, 2000, 2003, 2005; Kideckel, 1993; Torsello, 2003, entre otros). Este resurgimiento es lógico si consideramos que, como decíamos, la propiedad ha sido la piedra angular sobre la cual se han fundamentado los proyectos socialistas y liberales, socializándola en el primer caso y privatizándola en el segundo. Tanto en un caso como en el otro, las reformas en los sistemas de propiedad han sido consideradas esenciales para alcanzar los diferentes modelos de sociedad que se defendían. El caso que presentamos, el közbirtoksság, es también un ejemplo de un modelo de propiedad esencial para alcanzar el modelo de sociedad a la que pertenece. En este caso, no se trata de una sociedad liberal ni socialista, se trata de una sociedad eminentemente campesina organizada en una comunidad pequeña. Como veremos, esta institución condensa los principales valores y responsabilidades de una gobernanza local constituida a partir del parentesco y la vecindad.

Para el paradigma marxista, la propiedad privada de los medios de producción es la fuente de la explotación, y, por lo tanto, debe ser reemplazada por formas colectivas de propiedad. Por el contrario, los gobiernos post-socialistas, coincidiendo con gran parte de la comunidad internacional, consideraron la privatización como un elemento necesario que tenía que conducir a la economía de mercado y a la democracia. Desde esta última posición, la propiedad privada y el mercado del suelo agrícola y forestal es la única forma de generar incentivos para la productividad, la inversión, el acceso al crédito y el crecimiento, y es también la mejor manera de organizar las relaciones de propiedad de los recursos naturales y acabar con la corrupción. Estas eran, pues, las promesas del post-socialismo. En las últimas décadas, la antropología ha generado una visión crítica de estas 'promesas' a partir de diversas experiencias y de la crítica teórica a los planteamientos unilineales neoliberales ${ }^{4}$.

La realidad etnografiada de la Europa rural post-socialista muestra que esta privatización ha conducido, en la mayoría de los casos, a una agricultura de subsistencia y a grandes extensiones de tierra sin cultivar ${ }^{5}$. La des-colectivización condujo a la des-modernización de la agricultura. Los campesinos que lograron que fuese resti-

\footnotetext{
Hann (2006: 17-42), por ejemplo, parodiando precisamente el famoso The tragedy of the commons de Hardin, publica The tragedy of the Privates: Efficiency, Equity, and Justice in Postsocialist Euroasian Countryside, donde muestra la ineficiencia y desigualdad propias de los nuevos sistemas de privatización de la Europa postsocialista. Dorondel (2006) también cuestiona las teorías sobre los derechos de propiedad que enfatizan la efectividad de la propiedad privada para un uso sostenible y productivo de los recursos naturales como el bosque. Ver también Acheson (2015).

5 No podemos obviar que la devaluación de la agricultura, y la dificultad de los pequeños agricultores para subsistir, forma parte de un proceso global que se inició con la revolución verde y ha conducido a una sobreproducción agraria concentrada en pocas manos, a la desaparición de la explotación familiar y al éxodo de las zonas rurales. Ver por ejemplo Montagut y Dogliotti (2008: 99) y Dragos y Dabu (2003: 66).
} 
tuido su patrimonio familiar mostraron una tenaz resistencia a vender sus pequeñas parcelas, impidiendo la emergencia de un mercado de tierras y de una producción agrícola a gran escala. Esa tierra tiene una importancia ineludible para sus propietarios: económicamente, garantiza el derecho a la subsistencia y, moralmente, contiene el vínculo con el grupo de parentesco y con la comunidad ${ }^{6}$. Es decir, las condiciones bajo las cuales diferentes tipos de propiedad pueden ser alienadas en los mercados están sujetas a regulaciones morales además de legales. De este modo, la economía moral mantiene una presión constante que limita las posibilidades de una maximización "racional” impersonal (Hann, 2006: 39)7.

Mantenerse al margen de la modernización también es una forma de garantizar el derecho a la subsistencia y la autonomía ${ }^{8}$. En este contexto, "modernizarse" supone someterse a una dependencia respecto al precio de la gasolina, la inflación, la industria química o la tecnología. Algunos datos nos pueden dar una idea de la magnitud del riesgo que supone esta dependencia: entre 1995 y 1997 el herbicida Titus aumentó su precio diez veces, y en el año 2000 la gasolina había multiplicado por 30 y por 40 el precio de 1994 (Verdery, 2003: 207-208). Además, los casos de corrupción tanto a gran escala como a nivel más micro no cesaron de proliferar ${ }^{9}$.

Efectivamente, la privatización no condujo a un desarrollo económico tal y como lo entiende la economía capitalista, pero la restitución de la propiedad permitió a los vecinos de pequeñas sociedades rurales volver a ser comunidad y volver a ser campesinos $^{10}$. Maine (1965) ya destacó hace tiempo que las relaciones de propiedad son relaciones entre personas, luego en referencia a las cosas, y no relaciones entre las personas y las cosas (ver también Vinogradoff, 1913: 68; Gluckman, 1959). En el contexto estudiado, la recuperación de los derechos de propiedad permitió recuperar el vínculo entre los parientes muertos y los vivos contenidos en un determinado patrimonio familiar; les sirvió para adquirir, recuperar o perder estatus frente a la comunidad según la forma de trabajar la tierra y sus resultados; reforzó vínculos de comunidad a través de la asociación y la cooperación en relación de igualdad entre propietarios; fomentó un denso entramado de intercambios de bienes y servicios entre vecinos para poder realizar las tareas del campo sin asalariados, etc. En definitiva,

6 El vínculo del individuo/familia con la comunidad se articula mediante nociones como la identidad, el estilo de vida compartido o la reputación. Vemos, por ejemplo, que muchos pequeños propietarios siguen cultivando la tierra incluso si no es rentable porque su reputación como buenos campesinos está en juego en la interacción cotidiana de la comunidad.

A menudo esta economía moral se ha interpretado como una especie de irracionalidad o sentimentalismo campesino propio de otros tiempos que debía erradicarse en pro del progreso. Las palabras del ministro de agricultura rumano del año 1994 ejemplifican claramente esta posición: "For agriculture to develop, we must have an association of economic interest. Instead, we've generated a huge fund of emotions, and this means not evaluating land according to its intrinsic value. It becomes a talisman - "my father's hectare" and so forth — and people fight over this kind of thing" (citado en Verdery, 2003: 158).

8 En este contexto, la economía (la propiedad, la actividad productiva y el intercambio) se utiliza como un medio para el mantenimiento de lo social y, por lo tanto, está sometida al control de la comunidad. Es así como la riqueza súbita o excesiva despierta recelos y desconfianza. Foster (1965: 303) ya señalaba que a menudo la riqueza representa una amenaza para la cohesión social de las comunidades campesinas y Levi-Strauss (1969: 79) hablaba de que el miedo a ser envidiado puede inhibir el ejercicio de un privilegio.

$9 \quad$ Ver, por ejemplo, los grandes escándalos de desvíos y malversación de fondos que protagonizó Romcereal, la empresa estatal que debía proveer a los agricultores de pesticidas, herbicidas y fertilizantes, y distribuir créditos subvencionados (Verdery, 2003:110).

10 Ver Torrens (2006). 
la recuperación de los derechos de propiedad de la tierra, los animales, los pastos y el bosque sirve para ser campesinos, parientes, vecinos y miembros de la comunidad.

En este sentido el közbirtokosság es una institución coherente con muchas otras que organizan la vida social de la comunidad. La comunidad rural, en este caso, es una entidad socio-territorial con un alto grado de autosuficiencia y donde las unidades domésticas que la componen son altamente interdependientes y mantienen relaciones equitativas. Se trata de unidades sociales con una vida jurídica propia y local: "They were 'law generators' which had been proved over time by the fact of being preserved intact just like every work which meant to last. They never accepted the role of simple 'scope' of the normative acts designed by the deliberative structures of the modern state" (Rosculet, 2011: 149).

\section{Otras instituciones de asociación y cooperación de carácter comunal}

Antes de ahondar en el funcionamiento y la lógica del közbirtokosság veamos algunas otras instituciones que también son centrales para la vida local y que, como decía, son coherentes y conforman un todo que constituye un modelo de sociedad particular donde las formas de asociación y cooperación entre iguales están bien institucionalizadas.

Se trata de la organización en torno al ganado. Alrededor del $90 \%$ de explotaciones poseen menos de 5 hectáreas privadas (Instituto Nacional de Estadística 2010, en Sutcliffe et al. 2013: 60). Para estas explotaciones familiares la posibilidad de mantener ganado y sobrevivir como pequeños propietarios va ligada al acceso a los pastos comunales. Los rebaños pastan en tierras que se cultivan aprovechando que el estiércol es útil para la continuidad anual de los cultivos de cereales o patatas. Cada año cambian el lugar de los pastos para adaptarse al ciclo de cultivo y descanso de la tierra.

La mayoría de las familias tienen una o dos vacas, algunas pueden llegar hasta cinco, pero no más. Suelen tener unas cuantas ovejas y cabras, entre 5 y 10 . Este pequeño volumen de ganado también les obliga a asociarse para gestionar el trabajo que conlleva y conformar un único rebaño para todo el pueblo. El rebaño de vacas del pueblo cuenta con unas 200 vacas.

Cada año, antes de empezar la temporada de pasto, los hombres que quieren ser pastores deben someterse a una negociación y elección consensuada de todos los propietarios de vacas. Hacia el mes de marzo se reúnen todos en el centro del pueblo y los aspirantes a pastor exponen sus condiciones: cuánto y cómo quieren cobrar y qué ofrecen a cambio (experiencia, responsabilidad, juventud, conocimiento de las vacas, tradición familiar, etc.). Cuando cada uno de ellos ha expuesto su propuesta, los propietarios de las vacas discuten entre ellos y negocian con los futuros pastores hasta llegar a un acuerdo que se formaliza por escrito. Actualmente la competencia es escasa ya que, a causa de la despoblación y el envejecimiento de la población, no hay muchas familias que puedan permitirse el lujo de prescindir de uno de sus miembros adultos durante la temporada alta de trabajo agrícola. El acuerdo al que llegan, con pequeñas variaciones según el año, es el siguiente: los propietarios pagan al pastor $20 \mathrm{~kg}$ de maíz en grano y unos 2 euros por vaca y temporada de pastos. El pago se realiza al finalizar la temporada de pastos. Además, cada propietario dará al pastor una tarisznya (literalmente significa morral), el contenido y cantidad de la cual lo decide el propietario de acuerdo con su generosidad y con la satisfacción con 
el trabajo del pastor. Las tarisznya's acostumbran a contener huevos, panceta, carne, pálinka (aguardiente) y queso.

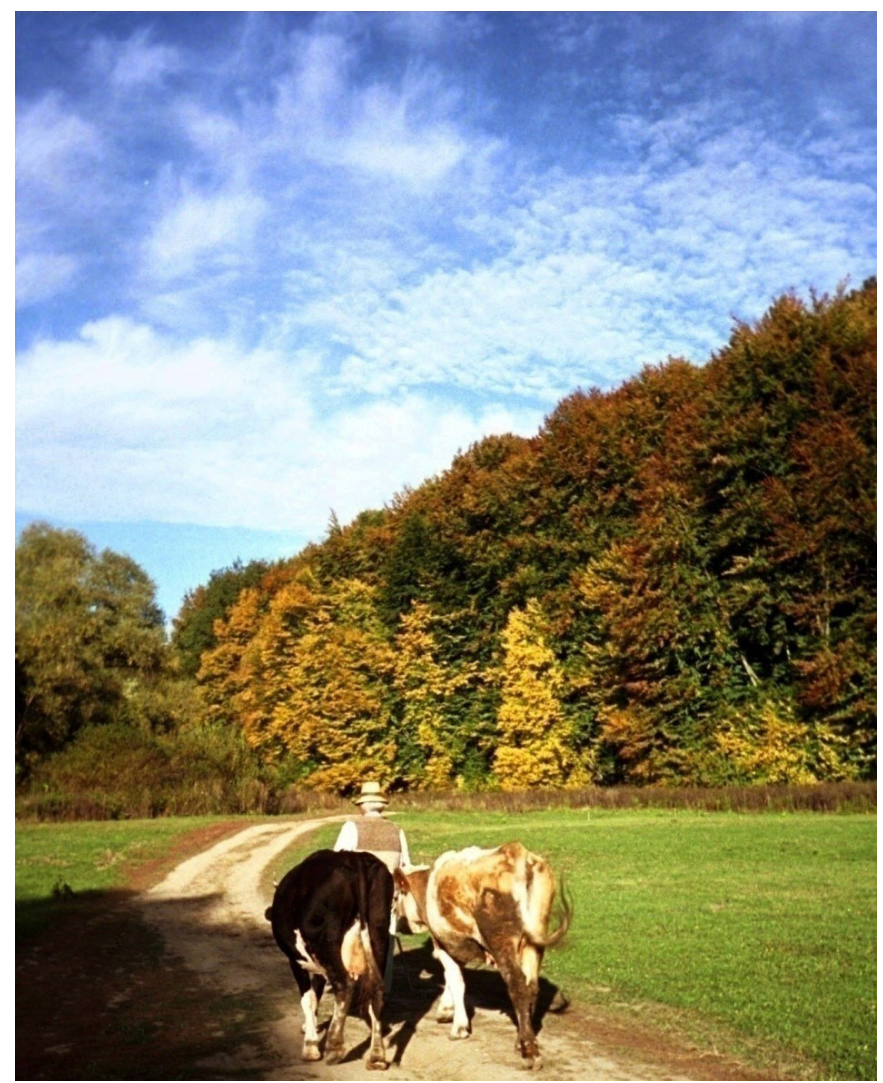

Fotografía 3. La mayoría de familias tiene una o dos vacas y unas cuantas ovejas

Vemos, pues, que los pagos están altamente desmonetizados. El acuerdo es esencialmente el reconocimiento de la interdependencia que existe entre los actores: campesinos y pastores. Y el precio viene marcado por la capacidad de producción y la necesidad de consumo de los actores evitando las fuertes discrepancias que se dan en economías de mercado entre los sacrificios de los actores implicados ${ }^{11}$.

Ser pastor del rebaño de ovejas (y algunas cabras) requiere otras habilidades, conocimientos y sacrificios. Las ovejas permanecen en los pastos de montaña desde abril hasta finales de noviembre, sin ir y venir del pueblo cada día como hacen las vacas. Por lo tanto, el pastor tiene que ordeñarlas, hacer queso y cuidar su salud durante todos estos meses. Y, además, protegerlas de los lobos durante la noche. $\mathrm{La}$ elección de pastor de ovejas es muy parecida a la del pastor de vacas. En este caso se reúnen en enero y el acuerdo siempre incluye una cláusula importarte que es la que

11 Appadurai (1986) habla de divergencia de valor cuando la lógica de los intercambios no mantiene relación con la magnitud de los sacrificios o el trabajo. 
fija las posibles compensaciones por daño o pérdidas de animales a consecuencia de ataques de lobos.

Un acuerdo razonable y habitual entre el pastor y los propietarios del ganado es el siguiente. Por cada oveja el pastor da a su propietario $8 \mathrm{~kg}$ de queso y un quilo y medio de ürda (queso fresco), por temporada. El resto del queso se lo queda él. Además, los propietarios deben pagar al pastor con 6 litros de trigo y unos 50 céntimos de euro por oveja y temporada. Ser pastor, tanto de ovejas como de vacas, es incompatible con muchos trabajos agrícolas y de crianza de otros animales. Es por ello que el pago al pastor con productos agrícolas compensa esta carencia.

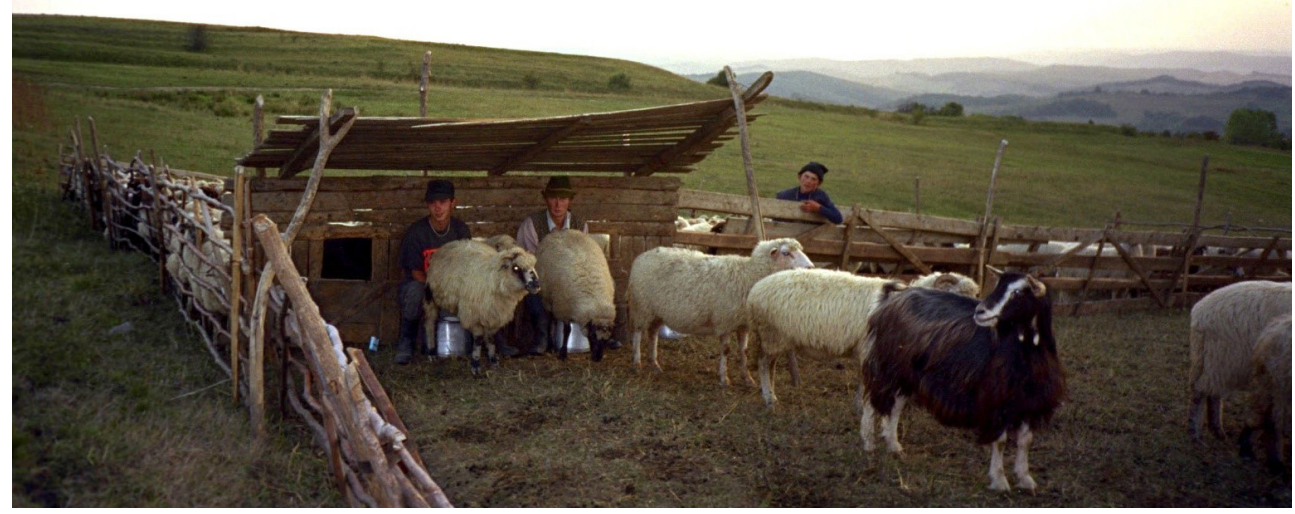

Fotografía 4. Ordeñando las ovejas

Una figura importante que hace de puente entre agricultores y pastores es el $\mathrm{Ma}$ jor biro que literalmente significa juez de campo. Su función es básicamente la de representar los intereses del colectivo de propietarios de ovejas y de los pastores, y la de mediar y testimoniar los conflictos que puedan surgir entre un propietario y el pastor. Por ejemplo, cuando una oveja da poca leche el pastor lo comunica al Major biro y éste al propietario. A finales de junio o principios de julio el propietario y el Major biro subirán al altiplano donde están los rebaños, a la hora de ordeñar las ovejas y, esta vez, ordeñarán la oveja en su presencia y medirán la leche en un pote de madera. Si la leche no llena el pote negociarán la cantidad de queso que el pastor deberá dar al propietario, y si la llena, el pastor deberá dar la cantidad acordada previamente. El Major biro debe someterse, como los pastores, a la elección del pueblo. La única compensación que recibirá es la leche de tres días de mayo de las ovejas y un poco de pálinka que algunos le darán voluntariamente.

Dos veces por temporada el Major biro organiza la jornada de desparasitación de las ovejas. Todas las familias que tienen ovejas tienen que enviar una persona como mínimo para trabajar. Como en el caso del Közmunka ${ }^{12}$, si los propietarios son dema-

12 Literalmente, trabajo en común. Se trata del trabajo no remunerado que realizan todos los miembros de la comunidad o como mínimo un representante de cada unidad doméstica, para realizar trabajos que benefician al 
siado viejos o están enfermos deben ser substituidos por alguien, ya sea un pariente o una persona pagada para la ocasión; si no hay parientes disponibles ni se dispone de recursos para pagar a alguien, deberán dar pálinka (aguardiente) para la celebración que sigue a la desparasitación.

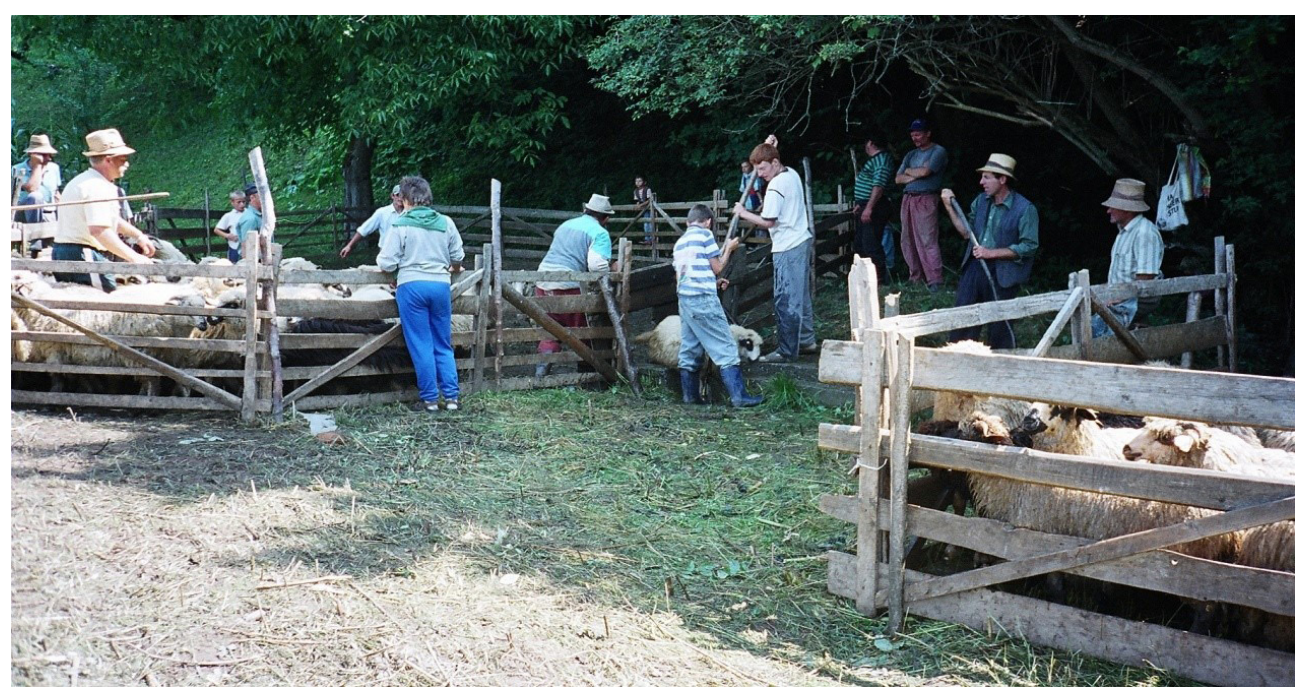

Fotografía 5. Desparasitando las ovejas

Este tipo de organización e instituciones lo encontramos con variaciones en la historia local de las comunidades rurales. Sutcliffe (2013: 63-64) describe para la región de Târnava Mare (sur de Transilvania) que el derecho a usar los pastos comunales hasta finales del siglo XIX se vinculaba al pago de una tasa por animal y a la contribución con determinados días de trabajo por animal para mantener los pastos. Las cuestiones relacionadas con los pastos se discutían y decidían en una asamblea anual pública presidida por el alcalde y el comité de pastos. Esto incluía elegir el pastor.

Vemos, pues, cómo la recuperación de la propiedad privada, en este caso del ganado, sirvió para recuperar instituciones asociativas y cooperativas que crean y recrean la comunidad con regulaciones locales propias. A continuación nos centramos en una de estas instituciones, közbirtoksság, que también contribuye a crear y re-crear la comunidad a través de la asunción de responsabilidades públicas y políticas con las ganancias obtenidas de la propiedad conjunta del bosque.

\section{El közbirtokosság: origen y tipologías}

El közbirtokosság es la institución propia de la minoría székely (húngara) de Transilvania que gestiona conjuntamente las propiedades forestales y los pastos de las co-

conjunto del pueblo, por ejemplo reparar caminos. Otra institución parecida es la kaláka: el trabajo no remunerado que vecinos, amigos y parientes de una persona realizan para ella, por ejemplo en la construcción de un establo o en una cosecha. 
munidades rurales. A pesar de que jurídicamente se considera una propiedad privada, se trata de una institución que no puede considerarse ni como propiedad privada ni pública y en la cual los derechos de propiedad y los derechos de uso no coinciden. La distinción histórica entre el derecho a representar la propiedad y el derecho a utilizarla fue bien reconocida por la ley de $1913^{13}$ de la antigua Hungría sobre la relación entre propiedad y propietario de campos, pastos y bosques del közbirtokosság. La mencionada ley establecía que el derecho de uso de las propiedades que forman el közbirtokosság pertenece al közbirtokosság y no al propietario. La ley, que reconocía la personalidad jurídica del közbirtokosság, cedía el derecho a los propietarios a vender o cambiar el territorio con permiso del Ministerio de Agricultura y con la mayoría de 2/3 partes de los votos de la asamblea de propietarios (Garda et alt. 2000).

En la evolución histórica del közbirtokosság podemos constatar la tensión entre los intereses del Estado, los intereses particulares de sus miembros, los intereses conjuntos de la mancomunidad de propietarios y los de la administración local. Son justamente los conflictos entre las diferentes unidades sociales que tienen derechos y deberes respecto a un mismo objeto, y que dan lugar a acuerdos, los que definen la propiedad en un periodo histórico y en un espacio concreto (Von Benda-Beckmann y Wiber 2006:15). Son conflictos que han dado lugar a una institución, cuyas regulaciones y funcionamiento han variado a lo largo de la historia ${ }^{14}$.

Actualmente el $50 \%$ de la superficie forestal de Rumania es propiedad del Estado y el otro $50 \%$ es de propiedad privada. El 58\% de esta superficie forestal privada (1,5 millones de hectáreas) es propiedad comunal gestionada de manera participativa. A diferencia de lo que ocurre actualmente en Europa occidental, también los pastos comunales mantienen un rol muy importante en Rumanía. Según el Instituto Nacional de Estadística (2010) se calcula que aproximadamente 3,4 millones de hectáreas de pastos permanentes en Rumanía se pueden considerar tierra comunal (Sutcliffe et alt. 2013: 59).

En este contexto, encontramos una amplia presencia de instituciones consuetudinarias de gestión comunal de bosques y pastos con variaciones locales y temporales. Algunas, por ejemplo, se basan en la residencia (región de Vrancea), otras en los derechos hereditarios, unas son más igualitarias y otras menos. Măntescu y Vasile (2009) describen tres tipologías básicas de propiedad comunal en Rumania: obştea, compossesorate y pădurea comunală. Según su clasificación, el közbirtokosság propio de Székelyföld pertenecería al tipo que en rumano se denomina compossesorate.

La obştea se encontraría en las regiones de Moldavia y Valaquia (especialmente en Vâlcea, Vrancea, Argeş, Gorj, Bacău y otros) y es el nombre con el cual las poblaciones designan la asamblea del pueblo, la propiedad comunal del pueblo y la institución local que gestiona la propiedad comunal del pueblo ${ }^{15}$. Hasta aquí coincide exactamente

13 En Valaquia y Moldavia, el código forestal de 1910 reconoció a las comunidades títulos legales de propiedad sobre los bosques que hasta el momento tomaban la forma de propiedades conjuntas instituidas consuetudinariamente (Măntescu y Vasile, 2009: 97). Ver también Rosculet (2011).

14 A partir de 1829, por ejemplo, la construcción de la red ferroviaria que daría acceso a los mercados centroeuropeos intensificó las presiones comerciales (Muica y Turnock, 2003: 9). Entre 1829 y 1922, tres millones de hectáreas fueron taladas reduciendo un 30\% las áreas forestales del país. Otras 1,3 millones de hectáreas fueron taladas entre 1922 y 1945. Después de la segunda guerra mundial Rumanía tuvo que pagar compensaciones de guerra a la Unión Soviética, y gran parte de esta deuda se pagó con leña para hacer funcionar la industria pesada (Măntescu y Vasile, 2009:97).

15 La denominación proviene del Eslavo y significa unión, corresponde a obshchina en ruso (Vasile, 2009) 
con el közbirtokosság ${ }^{16}$. Entre las diferentes obştea, Măntescu y Vasile (2009) distinguen las igualitarias de las no-igualitarias. En las primeras, propias de la región de Vrancea (al sur de Moldavia), todos los aldeanos tienen derecho a una parte igual de bosque y cada miembro tiene un voto en la asamblea que decide las cuestiones que afectan a la propiedad. La obştea funciona de manera participativa: se elige un comité ejecutivo, formado por 5-7 miembros, de los cuales uno es elegido presidente, y se rige por la asamblea del pueblo (Vasile, 2009). Cada miembro recibe anualmente la misma cantidad de leña para uso doméstico. El excedente de madera se vende y los beneficios se utilizan para mejoras de la comunidad: carreteras, canalización de agua, de gas, etc.

En el modelo no igualitario, no todas las personas del pueblo tienen el derecho al acceso a la propiedad comunal, sino que la propiedad se hereda de padres a hijos y el voto es proporcional al volumen de la propiedad. En este caso, los beneficios del excedente de madera que se vende se dividen entre los miembros de manera proporcional al volumen de la propiedad (Măntescu y Vasile, 2009). Como veremos, el közbirtokosság contiene elementos de los dos tipos: la leña para el consumo doméstico se reparte entre los hogares de forma equitativa; no se tiene en cuenta el número de personas de cada hogar ya que se necesita la misma cantidad de leña para cocinar y calentar una casa donde viven dos personas que una donde viven cuatro, me decían. Los beneficios obtenidos con la venta del excedente se invierten en mejoras para el pueblo, pero, en cambio, el volumen de las propiedades no es igual entre todos los miembros, y éstas se heredan. En todos los casos, la propiedad es indivisible. En la obştea existe solo un título de propiedad del bosque. Es, por tanto, una forma colectiva de propiedad forestal.

La segunda gran tipología que utiliza Măntescu es la compossesorate $^{17}$, propia de Transilvania y el norte de Rumanía (especialmente en el territorio que formaba parte del Imperio Austro-húngaro- Harghita, Covasna, Hunedoara, Arad, Brasov, Baia Mare y otros departamentos de Transilvania). Esta sería un tipo de propiedad conjunta muy similar a la obştea no-igualitaria. El közbirtokosság es la tipología de compossesorate que corresponde a la población székely. Encontramos elementos comunes con las obştea, pero es básicamente una propiedad conjunta, una asociación de propietarios. Se trata de una asociación muy particular regida por normas como la indivisibilidad y la inalienabilidad. Si la asociación decidiera disolverse, el bosque se convertiría en comunal entrando en el dominio público (Măntescu y Vasile, 2009: 103). Es, por tanto, un tipo de propiedad protegida y marginalizada del mercado.

Según Măntescu es un tipo de institución en la cual los co-propietarios se agrupan en unidades sociales que no se identifican ni con el pueblo ni con la comuna: "A compossessorate can result in grouping only a part or all the inhabitants of a village and it can also accept inhabitants of the neighbouring villages" (Caramelea, 1944, en Rosculet, 2010). El compossessorate regula la explotación conjunta de bosques y pastos, y los beneficios obtenidos en moneda y en leña se reparten de forma directamente proporcional al tamaño de la propiedad. A pesar de presentar esta tipología, Măntescu advierte de que no existe una sola forma de obştea ni de compossessorat y que las diferencias pueden ser sustantivas en las formas de gobernanza o distribución de beneficios (Vasile y Măntescu, 2009: 100). Veremos que en el caso etnografiado, efectivamente, se trata de una propiedad conjunta, con títulos de propiedad sepa-

16 Köz significa colectividad, comunidad; birtok significa propiedad, patrimonio, herencia, posesión (Eckhardt, Sándor y Konrád Miklós, 2003)

17 Compossesorate, igual que közbirtokosság, significa co-propiedad en rumano. 
rados y que se heredan, pero el reparto de leña anual para uso doméstico es igual para todos los miembros independientemente del volumen de su propiedad. Además, parte de los beneficios se invierte en mejoras para el pueblo. Es decir, que la falta de identificación de los co-propietarios con el pueblo que señala Măntescu tampoco se ajusta en absoluto con el caso etnografiado. Al contrario, veremos que es un instrumento importante de participación y gobernanza local.

La tercera tipología es la de pădurea comunală, donde la propiedad es del municipio rural, la gestiona el alcalde y todos los habitantes tienen igual derecho de acceso e igual derecho de cuota. En algunos casos los habitantes no perciben que tienen un derecho, sino más bien perciben la madera que proviene de la municipalidad como "ayuda", y la gente que realmente tiene acceso al bosque son las empresas forestales.

Esta sería pues una diferencia muy importante entre los modelos comunales y los modelos asociativos (tanto la obştea como el compossessorat) de gestión y propiedad del bosque. Los modelos asociativos otorgan más derechos y poder a los miembros que son conscientes de sus derechos y perciben la propiedad como privada. En cambio, en los modelos comunales, a pesar de que no es una propiedad estatal, la propiedad es privada y pertenece a la comuna, mucha gente percibe que el bosque es todavía del Estado y que las cuotas de madera que perciben son ayudas (Măntescu, 2009: 103). En este caso, los habitantes de la comuna no participan efectivamente de los procesos de toma de decisiones, más bien se limitan a presionar al alcalde para gestionar los recursos como consideran adecuado ${ }^{18}$.

Los pastos comunales que son propiedad de la comuna y son gestionados por el alcalde a menudo se denominan izlaz. El municipio alquila parcelas de pastos a los habitantes en función de sus necesidades o los destina a pastos comunales aplicando una tasa por animal (Sutcliffe, 2013). Generalmente, en el primer caso se trata del rebaño de ovejas (compuesto por las ovejas de todo el pueblo) y en el segundo de pasto para las vacas ${ }^{19}$.

\section{Historia reciente del közbirtokosság}

En el año 2000 el Estado devolvió la propiedad del közbirtokosság que había sido colectivizado durante el socialismo. La ley 1/2000 lo reconoció como institución legal y se restablecieron los antiguos estatutos consuetudinarios que defendían valores como la indivisibilidad, la inalienabilidad, la democracia participativa, la responsabilidad pública y el control de las desigualdades. Es decir, a pesar de que en ese momento el Estado ofrecía la libertad para modificar las regulaciones de cada közbirtokosság decididas por mayoría asamblearia, se restablecieron las regulacio-

18 En Transilvania y Bucovina muchas de estas formas comunales fueron el resultado del desmantelamiento de la frontera del Imperio Austro-húngaro. Según Măntescu (2009), el origen de estas propiedades es la donación que el emperador Josef II hizo a las poblaciones locales situadas en la frontera este del Imperio austrohúngaro a cambio de sus servicios militares. Mitrany (1930), en cambio, apunta que en Transilvania existían diversas formas de propiedades conjuntas como contraprestación a los servicios militares de sus habitantes pero sitúa su origen en el reinado de Maria Theresa (1740-80). La tierra que recibieron bajo esta forma fue gradualmente individualizada a través de diversas medidas legales mientras que los bosques y pastos permanecieron comunales. Otro tipo de propiedad tiene origen a finales de 1800: campesinos de una misma comunidad se asocian para el uso y la gestión de la propiedad pero mantienen el título de la propiedad separadamente. Y en Banat, las propiedades comunales obtenidas como reconocimiento de los servicios militares de los campesinos que defendían el Imperio Otomano toman el nombre de comunitate de avere (literalmente significa comunidad de la fortuna).

19 En el caso etnografiado sería diferente ya que también el rebaño de vacas agrupa a todas las vacas del pueblo. 
nes consuetudinarias antiguas. Măntescu (2009), siguiendo a Kingfisher y Maskovsky (2008), y refiriéndose al funcionamiento de las obştea, habla de indigenización del neoliberalismo, en la medida que los actores que participan en el Mercado son miembros de comunidades y grupos sociales que incorporan el sentido de obligaciones sociales en sus actividades económicas.

De hecho, inicialmente la ley que debía regular la restitución de los bosques preveía la devolución de los bosques a sus antiguos propietarios como propiedades privadas, individuales y separadas con límites físicos reales. El problema es que toda la historia de las cooperativas forestales y las propiedades conjuntas del bosque fueron difuminando en algunos casos los límites reales (físicos) entre las diferentes parcelas que las componían. Las diferentes propiedades del bosque se convirtieron, pues, en una cuota, una proporción del conjunto de la propiedad del közbirtokosság ${ }^{20}$. Por lo tanto, finalmente el Estado restituyó la propiedad de estos bosques como dividendos de un conjunto de propiedades conjuntas o comunales. Aún así, en ese momento ofrecía la posibilidad de dividir el közbirtokosság en parcelas individuales entre sus propietarios. En la comunidad estudiada, como en tantos otros lugares, se decidió por votación restablecer el közbirtokosság.

En este sentido, es muy interesante contrastar diferentes casos etnográficos en los cuales la restitución de la propiedad forestal se produjo de formas muy diferentes. Dorondel (2009: 50) presenta un caso, en Dragomireşti, en el cual todos los habitantes recibieron 10 hectáreas de bosque: "While they had nominal rights to property, however, the forest owners did not acquire effective access to their forest. Instead, patron-client relationship between government officials and Rudari undermined the position of private owners and buttressed the power of local state officials". Todo ello condujo a la reproducción de unas relaciones asimétricas y de explotación y a una deforestación muy importante a partir de 2003. Măntescu y Vasile (2009: 105), en cambio, exponen el caso de la comuna de Stulpicani, en el condado de Suceava, donde antes del socialismo el bosque era comunal y en el proceso de restitución de la propiedad uno de sus pueblos, Gemenea, logró transformar este bosque comunal en una obştea de 783 hectáreas basada en un principio de herencia ${ }^{21}$. En este caso no se trataba, pues, de una forma restaurada sino más bien "inventada" o "tomada prestada" con la finalidad de mantener la propiedad en manos de la comunidad.

Esta es una buena opción para un tipo de explotación tan particular como es el bosque. Una parte importante de los derechos y deberes asociados a la propiedad del bosque es controlada por el Estado. La ley garantiza la regeneración del bosque, el desarrollo sostenible, establece los requerimientos de reforestación, la madera que puede ser talada en un periodo determinado, etc. Estas regulaciones y la propia naturaleza del bosque como recurso natural, hace que la gestión comunal o conjunta sea la opción más racional para su explotación. Así, a diferencia de otras explotaciones más inten-

20 En este sentido Măntescu y Vasile (2009:101) dicen que tanto la obştea como el composesorat no pueden considerarse una asociación de propietarios ya que las cuotas que uno posee no corresponden a parcelas de bosque delimitadas que se ponen juntas sino que corresponde a la cantidad de producto que se puede obtener del bosque y al número de votos en la asamblea general.

21 Ello se logró gracias al empeño de una mujer del pueblo que reivindicó y trató de demostrar ante el juez que antes de 1948 el bosque comunal estuvo a punto de transformarse, o se transformó, en obstea. Parece que el éxito se debió más a la buena voluntad del juez y a la red de relaciones personales de esta mujer, que a la consistencia de sus pruebas. Cuando se consiguió el reconocimiento de la obştea siguieron los conflictos de abusos de poder y clientelismo que promovía esta persona. 
sivas como las agrarias, la explotación del bosque se ha gestionado conjuntamente en muchos momentos históricos, en diferentes espacios geográficos y en diferentes contextos políticos y económicos. Además de estas restricciones y obligaciones respecto a la propiedad del bosque controladas por el Estado, una característica importante de su explotación es su horizonte temporal. Mientras que la agricultura, por ejemplo, tiene como mínimo una cosecha al año, en el caso de los árboles normalmente se pueden talar a partir de los 50 años o más. Mientras tanto, el bosque requiere de una inversión notable para protegerlo y limpiarlo. Todo ello hace que el sistema más racional de explotación y gestión del bosque sea a gran escala. La pluriocupación a pequeña escala en la agricultura y la ganadería, la organización de la fuerza de trabajo básicamente entre los miembros de la familia y el hecho de ser mayoritariamente pequeños propietarios, hace que los requerimientos de la gestión y explotación del bosque supere las posibilidades de sus propietarios. Por lo tanto, la asociación de estos pequeños propietarios para explotar conjuntamente el bosque es la única opción para subsistir frente a las grandes empresas forestales (Devillard, 1996).

\section{Reglas de equidad y conflictos en el közbirtoksság}

Veamos cuáles son las regulaciones del közbirtokosság. Actualmente, en Siklód todos los propietarios del közbirtokosság son miembros de la asamblea general, que es donde se toman las decisiones mediante votaciones: se decide el comité que ejecutará la gestión, la aceptación de nuevos miembros, la cantidad de madera que se venderá, la cantidad que se distribuirá entre los miembros, las inversiones, etc. Cada miembro representa un voto en la asamblea. Un propietario no puede poseer más de un 5\% de la propiedad total del közbirtokosság. La propiedad solo puede alienarse entre los miembros del közbirtokosság y, si más de un propietario está interesado en comprar una misma parcela, tienen que repartirse la propiedad, no pueden competir en precios. Si el közbirtokosság adquiere nuevas propiedades estas son repartidas entre todos los miembros. De esta forma se limitan las posibles desigualdades tanto materiales como en la toma de decisiones garantizando el acceso a los recursos y a la participación de todos los miembros.

En cuanto a los beneficios, aproximadamente un $25 \%$ se invierte en mejoras para el pueblo y otro $25 \%$ se destina al consumo doméstico. El resto se reparte entre inversiones, salarios del comité, y se separa un remanente para poder reaccionar en caso de catástrofes naturales. En el año 2003, por ejemplo, se decidió invertir parte de los beneficios en mejorar las calles del pueblo y hacer llegar agua corriente a todas las casas. Si el dinero necesario para todas estas mejoras se obtuvo del közbirtokosság, la mano de obra necesaria se obtuvo mediante otra institución consuetudinaria: el közmunka. Como se ha explicado antes, se trata del trabajo no remunerado de todos los habitantes del pueblo o, como mínimo, de un representante de cada unidad doméstica para realizar tareas que beneficien al conjunto del pueblo. Si la salud o la edad de alguien no le permite realizar el trabajo, deberá procurarse de una persona que le substituya. Si no hay ningún vecino, pariente o amigo que le pueda substituir se acostumbra a pagar a alguna persona para que lo haga. Algunas personas, si se lo pueden permitir, pagan a más de una persona para colaborar con el proyecto comunitario. Un anciano, por ejemplo, contrató a dos hombres para trabajar dos días, pagaba 200.000 leis por hombre y día, más pálinka, café y comida. Este es un gesto que otorga prestigio a quien lo hace, implica generosidad, compromiso comunitario 
y medios. De esta forma, el közbirtokosság se define como una institución de propiedades privadas con responsabilidades públicas.

Lógicamente, todo ello no transcurre sin conflictos o desacuerdos. En la reparación de las calles del pueblo, algunas personas mostraban su desacuerdo en tener que reparar caminos alejados del centro, donde vivía únicamente alguna anciana y, por lo tanto, ella era la única que se beneficiaría de la inversión. Otro conflicto importante surgió cuando el Pastor religioso del pueblo, que había sido elegido democráticamente en asamblea para dirigir la gestión del közbirtokosság, fue acusado de malversar los fondos ${ }^{22}$. En esta ocasión no sólo tuvo que abandonar el cargo, sino que tuvo que solicitar otro destino para ejercer como Pastor.

Otra discusión versaba sobre si todas las unidades domésticas del pueblo debían recibir la misma cantidad de leña para uso doméstico o recibir una cantidad proporcional al volumen de la propiedad de cada una. Finalmente se decidió que todas las unidades domésticas recibieran la misma cantidad de leña para uso doméstico independientemente del volumen de su propiedad. Por lo tanto, se distingue entre lo que es una necesidad básica que se entiende como un derecho y lo que son las ganancias que se reciben en proporción al volumen de la propiedad. En el año 2003, todos los beneficios se destinaron a arreglar las calles del pueblo y cada unidad doméstica recibió 8 metros de madera (se cuenta como 8 metros de largo y 1 metro de alto) destinada al uso doméstico. Se considera una cantidad suficiente para pasar un invierno en un entorno donde la leña constituye el único sistema de combustión para cocinar y calentarse y en donde los inviernos las temperaturas pueden llegar a descender hasta los $-25^{\circ} \mathrm{C}$.

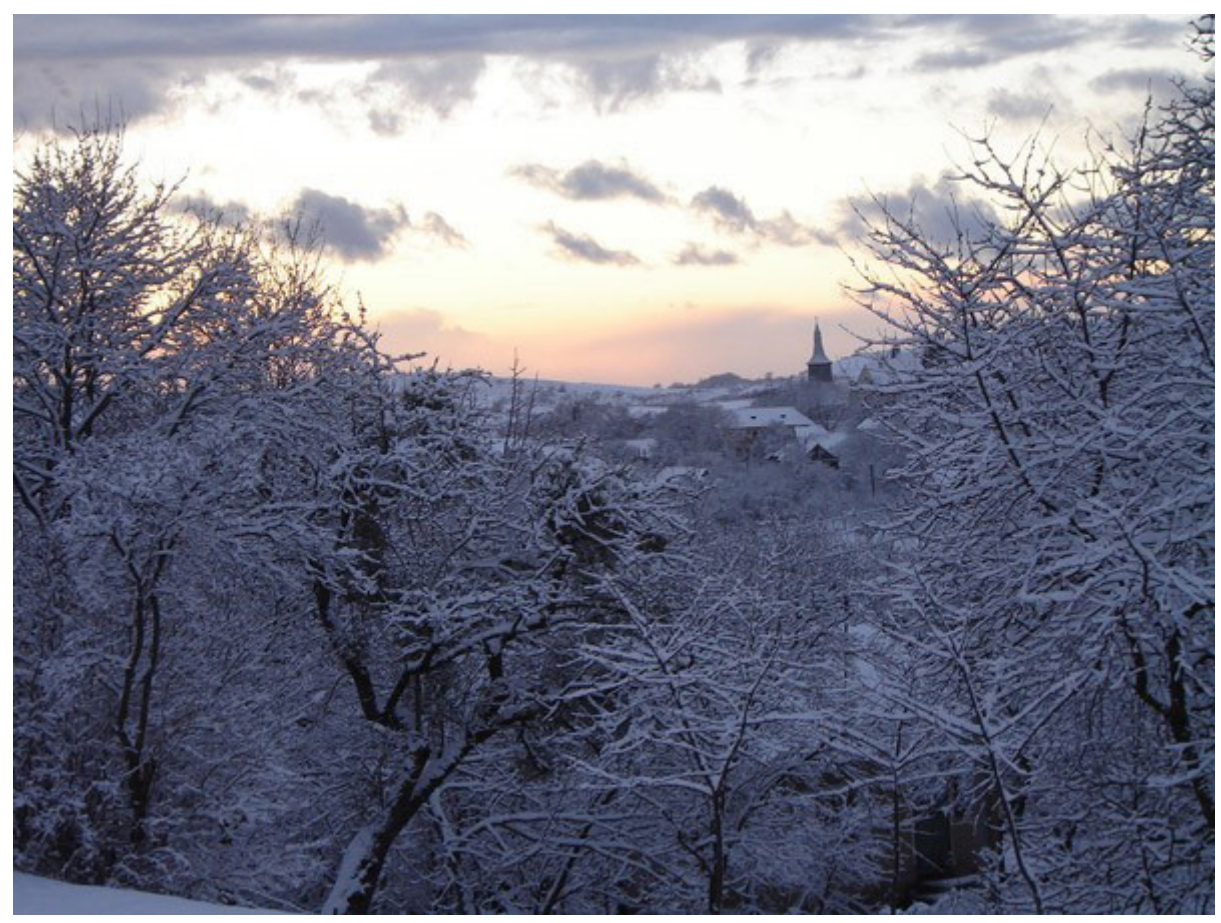

Fotografía 6. En invierno las temperaturas pueden descender a $-25^{\circ} \mathrm{C}$

22 Para ahondar en el fenómeno de la corrupción de las asociaciones forestales ver Vasile (2009). 


\section{Conclusiones}

La mirada que proponemos cuando pensamos en los derechos de propiedad es la que formula la pregunta de Kligman y Verdery (2011:8): "how persons are made through their relation to things". El közbirtoksság nos parece un buen ejemplo para re-pensar la propiedad abandonando la dicotomía público/privado, y entendiéndola como un conjunto de derechos sobre las cosas que pueden ser ejercidos por diferentes personas e instituciones en relación al mismo objeto, destacando funciones políticas y sociales además de las económicas. Es un buen ejemplo de una forma de propiedad en la cual lo público y lo privado aparecen inextricablemente mezclados, y donde la distinción entre el derecho de representar una propiedad y los derechos de utilizarla y explotarla económicamente es clara.

En este caso parece que efectivamente las funciones sociales, de responsabilidad pública y comunitaria son prioritarias para el funcionamiento del közbirtokosság. Mediante la propiedad se defiende el derecho al acceso igualitario de los recursos naturales necesarios para la subsistencia. Es un tipo de propiedad sujeta a regulaciones morales además de legales y económicas. La propiedad conjunta del közbirtokosság supera de lejos el valor de las sumas de las propiedades. Esta propiedad conjunta da un valor y una función al individuo en comunidad, al individuo en sociedad, tanto en lo referente a la toma de decisiones (voto en la asamblea independientemente del volumen de la propiedad) como en lo referente a las necesidades (cantidad de leña para uso doméstico recibida). Vemos también cómo entre sus regulaciones destacan aquellas en las que el objetivo es el de limitar las desigualdades.

Otro aspecto que me parece importante es que la propiedad no solo es un derecho, es sobre todo una obligación responsable para/con la comunidad. En este caso, es claro que los derechos y los deberes de los miembros del közbirtokosság superan los asuntos relacionados con la propiedad del bosque y se relacionan con la gobernanza local. Si antes decíamos que contemplaba y asumía la responsabilidad de las necesidades individuales, también asume las necesidades comunitarias como la mejora de infraestructuras comunes (agua, caminos, etc.). La lógica de su funcionamiento prioriza aspectos sociales básicos y responsabilidades públicas al tiempo que respeta y reconoce una diferenciación interna (no todos los miembros poseen el mismo volumen de propiedad ni obtienen los mismos beneficios).

En definitiva, hemos querido mostrar cómo, al margen de su función económica, el közbirtokosság tiene una función social y pública importante en la medida que define un modelo de sociedad y se implica en la gestión de los asuntos públicos.

\section{Bibliografia}

Acheson, James (2000). "Clearcutting Maine: Implications for the Theory of Common Property Resources”. Human Ecology, 28 (2): 145-169.

- (2015). "Private land and common oceans. Analysis of the development of property regimes". Current Anthropology, 56 (1): 28-55.

Catwright, Andrew (2000a). "Against 'Decollectivisation': Land Reform in Romania, 19901992 Perspective". Working Paper n4, Halle/Saale: Max Planck Institute for Social Anthropology. 
- (2000b). "State law and the recognitin of property in rural Romania". Working Paper $\mathrm{n}^{\circ} 10$, Halle/Saale: Max Planck Institute for Social Anthropology.

- (2003). "Private Farming in Romania: What are the old people going to do with their land?" en Hann, Chris (Ed.), The Postsocialist Agrarian Question. Max Planck Institute for Social Anthropology. Münster: Lit Verlag, 171-188.

- (2005). "The security of properties in Rumania", en Hann, Chris (Ed.), Property Relations: the Halle Focus Group, 2000-2005. Halle: Max Planck Institute for Social Anthropology.

Creed, Gerald W. (1998). Domesticating Revolution. From Socialist Reform to Ambivalent Transition in a Bulgarian Village. Pennsylvania: The Pennsylvania State University Press.

Devillard, Marie José (1996). "La construcción social de los usos colectivos agrarios", en Chamoux, Marie Noëlle y Contreras, Jesús (1996) La gestión comunal de recursos. Barcelona: Icaria.

Dorondel, Stefan (2006). "Common forest, private benefits: access to state and politics in a village in postsocialist Romania". IASCP Europe Regional Meeting. Building the European Commons: from open fields to open source. Brescia-Italy March 23-25.

- (2009). “"They should be killed' Forest restitution, ethnic groups and patronage in postsocialist Romania", en Fay, Derick; James, Deborah (Eds.) The Rights and Wrongs of Land Restitution. New York: Routledge, 43-65.

Dragos, Paul; Dabu, Adina (2003). "Land Reform and Agricultural Reform Policies in Romania's Transition to the Market Economy". Eastern European Economics, 41 (5): 49-69.

Foster, George, M. (1965). "Peasant Society and the Image of Limited Good". American Anthropologist, 67 (2): 293-315.

Garda; Birtalan; Ráduly; Tamás (2000). Tájékoztató és Gyakorlati Útmutató. Sepsiszentgyörgy: T3 Info Kiadó

Gluckman, Max (1959). "The technical vocabulary of Barotse". American Anthropologist, 61 (5): 743-759.

Hann, Chris (2005). "Main theme: Property, Neoliberalism and Rural Privatization" en Chris Hann (Ed.), Property Relations: the Halle Focus Group, 2000-2005. Max Planck Institute for Social Anthropology, Halle/Saale.

Hann, Chris (Ed.)

- (2003). The Postsocialist Agrarian Question. Max Planck Institute for Social Anthropology, Münster: Lit Verlag.

- (2006). Not the horse we wanted! Postsocialism, neoliberalism, and Euroasia, Münster: Lit Verlag.

Kaneff, Deema (2000). "Property, Work and local identity". Working Paper n⿳015, Halle/ Saale: Max Planck Institute for Social Antropology.

Kideckel, David A. (1993). The Solitude of Collectivism. Romanian Villagers to the Revolution and Beyond. New York: Cornell University.

Kligman, Gail; Verdery, Katherine (2011). Peasants under siege. Princeton: Princeton University Press.

Lévi-Strauss, Claude (1969). Las estructuras elementales del parentesco. Barcelona: Paidós. Maine, Henry (1965 [1861]). Ancient Law, London: Dent.

Măntescu; Vasile (2009). "Property reforms in rural Romania and community-based forest". Romanian Sociology (Sociologie Romaneasca), 7 (2): 95-113.

Măntescu, Liviu (2009). "When Globalization meets Postsocialism. Community-based institutions of timber market in Romania". Presentado en el seminario de la facultad de economía de la Universidad de Navarra. 
Mitrany, David (1930). The Land and the peasant in Rumania: the war and the agrarian reform, 1917-1921. London: Oxford University Press.

Montagut, Xavier; Dogliotti, Fabrizio (2008). Alimentos Globalizados. Icaria: Barcelona.

Roşculet, Gheorghe (2010). "The compossessorates in Olt County". Bulletin of the Transilvania University of Braşov, 3 (52): 57-62.

- (2011). "The forest code of 1910 applied to the forests belonging to the compossessorates and the rural communities". Bulletin of the Transilvania Univerity of Braşov, 4 (53) No.2: 147-155.

Sutcliffe, Laura et al. (2013). "Pastoral commons use in Romania and the role of the Common Agricultural Policy". International Journal of the Commons, 7 (I): 58-72.

Scott, James (1976). The Moral Economy of the Peasant. Rebellion and Subsistence in Southeast Asia. New Haven and London: Yale University Press.

Torsello, Davide (2003). Trust, Property and Social Change in a Southern Slovakian Village. Münster: Lit Verlag.

Torrens, Míriam (2006). “Individuo, familia y comunidad. Un estudio de caso en Transilvania”. Perifèria, 4: 1-22.

-(2013). Pagesos del Post-socialisme. Un estudi de comunitat a Transilvània. Tesis doctoral. Departamento de Antropología Social y Cultural. Universidad Autónoma de Barcelona.

Vasile, Monica (2007). "Decentralization as an aid to enhancing corruption in Romanian forest". Conference of American Anthropological Association in November 2007, Washington DC.

- (2009). "Corruption in Romanian forestry-morality and local practice in the context of privatization”. Revista Română de Sociologie, serie nouă, anul XX, nº1-2: 105-120.

Verdery, Katherine (2001). "Inequality as temporal process. Property and time in Transylvanian's land restitution”. Anthropological Theory, 1: 373-392.

- (2003). The Vanishing Hectare. Property and value in postsocialist transylvania. New York: Cornell University.

Vinogradoff, Paul (1913). Common sense in law. London: Williams

Von Benda-Beckmann, Franz; Von Benda-Beckmann Keebet; Melanie G. Wiber (2006). "The properties of property" en Von Benda-Beckmann Franz and Von Benda-Beckmann Keebet; Melanie G. Wiber (Eds.), Changing Properties of Property. Oxford-New York: Berghahn books: 1-39. 